\title{
Stability Analysis for Pod Yield and Its Component Traits in Some Peanut Genotypes
}

\author{
Ghada B. Abd El-aziz ${ }^{1}$ and Hoda. E.A. Ibrahim ${ }^{2}$ \\ ${ }^{1}$ Oil Crops Dep., FCRI, A R C, Giza, Egypt \\ ${ }^{2}$ Central Laboratory for Design \& Statistical Analysis Research, A R C, Giza, Egypt. \\ Corresponding author: darshahmed21@gmail.com
}

\begin{abstract}
Eight peanut genotypes were grown at two locations during four successive summer seasons from 2014 to 2017 to give eight environments in order to evaluate yield stability. Significant genotype $\times$ environment interaction was detected for all traits. Results revealed that peanut genotypes Sohag116, Sohag119 and Sohag120 were superior in their mean performance for yield and yield components, The regression coefficient value was approached unity in genotypes VAC-R92, Sohag nos. 116, 119 and 120 also, pod weight and seed weight, genotypes Sohag nos. 116 and 119 as well as number of pods and number of seeds plant ${ }^{-1}$ genotypes Giza 6, Line 9, VAC-R92 and Sohag 119 as well as shelling percentage, genotypes Sohag 112, Line 9, Introduction 508 and Sohag 120 as well as 100-pod weight, genotypes Sohag 112, Introduction 508 and VACR92 for 100-seed weight genotypes Sohag 112, Line 9, Introduction 508 and Sohag 116 for pod yield fed ${ }^{-1}$., where the value of bi almost approached unity, indicating average response to the fluctuating environmental conditions prevailed. Genotypes VAC-R92, Sohag nos. 116 and 120 had the highest pod weight plant ${ }^{-1}$, number of pods plant ${ }^{-1}$, number of seeds plant ${ }^{-1}$ and seed weight plant ${ }^{-1}$ among the tested genotypes, as they had high mean of pods (50.19) over population average mean of pods plant ${ }^{-1}$ (46.5) peanut genotypes VAC-R92, Sohag nos. 119 and 120 for 100- pod weight (g), genotypes Sohag nos. 116 and 119 for 100-seed weight (g), genotypes Sohag nos. 116, 19 and 120 for pod yield fed $^{-1}$ (ard.). These genotypes are suitable especially for favorable growing seasons as they had nearest (bi) value to 1. genotype Sohg112 recorded the highest number of pods plant $^{-1}$ over the grand mean, whereas genotypes Sohag nos. 116 and 120 gave highest shelling percentage, genotype Sohag116 gave the highest 100-pod weight and Sohag120 gave the highest number of seeds plant ${ }^{-1}$ indicated that these genotypes are fitted, for less favorable locations as they had low (bi) value (b<1). Such genotypes can be utilized in a breeding program for transferring stability characters in to high yielding cultivars peanut as genotype 8 which was the best one.
\end{abstract}

Key words: Peanut, Stability, Pod yield, Yield components.

\section{Introduction}

Groundnut or peanut (Arachis hypogaea L.), a segmental allopolyploid, self-pollinated legume. Popularly known as peanut, groundnut or poor man's cashew. It is widely cultivated as legume/oil crop in more than 114 countries including tropical to temperate region (Abo-Elezz et al., 2010). It is an important oil, food and feed legume, where kernels are rich in oil (48-50\%) and protein $(25-28 \%)$. It stated that global groundnut production increased marginally in last decade by just $0.4 \%$ only (Janila et al., 2013). Since Asian and African countries accounts for the $93 \%$ of global groundnut production, where cultivation is predominantly under rainfed and resource poor conditions (Knauft and Gorbet, 1993). The lower productivity in groundnut is mainly due to various biotic and abiotic stresses. Yield is a complex character resulting from interplay of various yield contributing characters, which have positive or negative association with yield and among themselves also. The consistent performance of a genotype over a range of environments is essential for a wide stability of a variety. Stability of genotypes depends upon maintaining expression of certain morphological and physiological attributes and allowing others to vary, resulting in $\mathrm{G} \times \mathrm{E}$ interactions. $\mathrm{G} \times \mathrm{E}$ interaction has a masking effect on the performance of a genotype and hence the relative ranking of the genotype do not remain the same over number of environments. Stability of genotypes to environmental fluctuations is important for stabilization of crop production both temporally and spatially. Estimation of phenotypic stability, which involves regression analysis, has proven to be a valuable tool in the assessment of varietal adaptability. Stability analysis is useful in the identification of stable genotypes and in predicting the responses of various genotypes over changing environments (Eberhart and Russell, 1966; Finlay and Wilkinson, 1963). It is generally agreed that the more stable genotypes adjust their phenotypic responses to provide some measure of uniformity in spite of environmental fluctuations (Patil et al., 2014). Therefore, an attempt has been made in present study to evaluate different groundnut genotypes across the different locations to know the role of $\mathrm{G} \times \mathrm{E}$ interactions and also to analyze the stability of genotypes for different traits.

\section{Materials and Methods}

The experiments were carried out during four successive summer seasons of 2014, 2015, 2016 and 
2017 at Assuit and Shandweel agricultural research station. Eight peanut genotypes were used for this experiment. The name and origin of genotypes are shown in Table (1). 6 Soil samples were collected from each experimental area (Ass., Sh.) from the upper soil layer $(30 \mathrm{~cm})$. The samples from each experimental area were mixed together to make combined sample for each location. Each combined samples was subjected to lab analysis to determined physical and chemical properties of soil as presented in Table (2). The experiment was laid out in a randomized complete block design (RCBD) with three replications at eight environments (2 locations x 4 years). Plot area was $9.6 \mathrm{~m}^{2}$ (4 rows, $4 \mathrm{~m}$ long and $60 \mathrm{~cm}$ apart). Distance between hills within rows was $15 \mathrm{~cm}$ with one plant left per hill after thinning. Cultural practices were done according to recommendations. The two guarded inner rows were harvested to determine the following characteristics: pod weight plant $^{-1}(\mathrm{~g})$, number of pods plant ${ }^{-1}$, number of seeds plant ${ }^{-1}$, seed weight plant $^{-1}(\mathrm{~g})$, shelling percentage $(\%), 100$-seed weight $(\mathrm{g}), 100$ pod weight $(\mathrm{g})$ and pod yield $\mathrm{fed}^{-1}$. (ardab), where (one $\operatorname{ardab}=75 \mathrm{~kg}$ and one feddan $=4200 \mathrm{~m}^{2}$ ). Data of yield components were recorded on ten guarded plants per plot.

Table 1. Name and Origin of the eight peanut genotypes.

\begin{tabular}{llll}
\hline No & \multicolumn{1}{c}{ Genotype } & \multicolumn{1}{c}{ Pedigree } & \multicolumn{1}{c}{ Origin } \\
\hline 1 & Giza6 (G1) & A commercial cultivar & Egypt \\
2 & Sohag112 $(\mathrm{G} 2)$ & A line selected from H7 x VAC-R92 & Egypt 1998 \\
3 & Line9 (G3) & A line selected from L 382 x Giza5 & Egypt \\
4 & Introduction 508 (G4) & Not available & USA \\
5 & VAC-R92 (G5) & Not available & USA \\
6 & Sohag116 (G6) & A line selected from H9 x NC-7 & Egypt 1998 \\
7 & Sohag119 (G7) & A line selected from Intr.500 x L262 & Egypt 1998 \\
8 & Sohag120 (G8) & A line selected from Intr.500 x NC-7 & Egypt 1998 \\
\hline
\end{tabular}

Table 2. Some physical and chemical properties of experimental soils of Assuit (Ass.) and Shandweel (Sh).

\begin{tabular}{|c|c|c|c|c|c|c|c|c|c|c|c|c|c|c|c|c|}
\hline \multirow{3}{*}{ Years } & \multirow{2}{*}{\multicolumn{2}{|c|}{ Texture }} & \multirow{2}{*}{\multicolumn{2}{|c|}{$\mathrm{Ca}^{++}$}} & \multirow{2}{*}{\multicolumn{2}{|c|}{ EC dsm }} & \multirow{2}{*}{\multicolumn{2}{|c|}{ Soil ph }} & \multirow{2}{*}{\multicolumn{2}{|c|}{$\begin{array}{c}\text { Organic } \\
\text { matter } \\
(\text { O.M) }\end{array}$}} & \multicolumn{6}{|c|}{ Available nutrients in soil (ppm) } \\
\hline & & & & & & & & & & & \multicolumn{2}{|c|}{$\mathbf{N}$} & \multicolumn{2}{|c|}{$\mathbf{P}$} & \multicolumn{2}{|c|}{ K } \\
\hline & Ass. & Sh. & Ass. & Sh. & Ass. & Sh. & Ass. & Sh. & Ass. & Sh. & Ass. & Sh. & Ass. & Sh. & Ass. & Sh. \\
\hline 2014 & sandy & $\begin{array}{l}\text { Clay } \\
\text { loam }\end{array}$ & 2.16 & 7.6 & 0.42 & 0.087 & 8.10 & 7.8 & 0.27 & 1.1 & 0.5 & 15 & 8.31 & 18 & 11.7 & 82 \\
\hline 2015 & sandy & $\begin{array}{l}\text { Clay } \\
\text { loam }\end{array}$ & 2.10 & 7.9 & 0.39 & 0.09 & 8.50 & 7.9 & 0.22 & 1.3 & 0.3 & 18 & 8.32 & 19 & 11.9 & 77 \\
\hline 2016 & sandy & $\begin{array}{l}\text { Clay } \\
\text { loam }\end{array}$ & 2.00 & 7.8 & 0.35 & 0.086 & 8.55 & 7.7 & 0.21 & 1.1 & 0.4 & 16 & 8.28 & 19 & 12.1 & 80 \\
\hline 2017 & sandy & $\begin{array}{l}\text { Clay } \\
\text { loam }\end{array}$ & 2.18 & 7.7 & 0.40 & 0.089 & 8.47 & 7.9 & 0.25 & 1.2 & 0.4 & 17 & 8.30 & 18 & 12.0 & 79 \\
\hline
\end{tabular}

Homogeneity test was used to satisfy the assumption of homogeneity of variances before running the combined analysis on the eight genotypes and eight environments (two locations and four years) according to Bartlett's test.

A combined analysis of variance across locations was computed assuming replications and locations effects as random and genotypes as fixed variable (Steel et al., 1997). Mean comparisons for these traits were done according to Duncan's Test at P < 0.05 (Duncan, 1955).

\section{Stability analysis}

The stability analysis was done following Eberhart and Russel (1966) model which interprets the variance of regression deviations as a measure of cultivar stability and the liner regression coefficient (b) as a measure of environmental index. In this model, mean $(\mu)$ and environmental index (Ij) are used as dependent and independent variables respectively to compute the regression coefficient.
According to this model, an ideal genotype should have high mean $(\mu>X)$, a unit regression coefficient $(\mathrm{bi}=1)$ and no deviation from linearity $\left(\mathrm{S}^{2} \mathrm{di}=0\right)$.

The basic model for the Eberhart and Russel (1966) model is:

Where,

$$
Y i j=\mu i+\beta i I j+\delta i j,
$$

$\mathrm{Y}_{\mathrm{ij}}=$ genotypic mean of $\mathrm{i}^{\text {th }}$ genotype at $\mathrm{j}^{\text {th }}$ environment. $\mu \mathrm{i}=$ mean of $\mathrm{i}^{\text {th }}$ genotype over all environments $\mathrm{bi}=$ regression coefficient which measures the response of $\mathrm{i}^{\text {th }}$ genotype to environments $I_{j}=$ environmental index as mean of all genotypes at $\mathrm{j}^{\text {th }}$ environment minus the overall mean, and $\delta \mathrm{ij}=$ deviation from regression coefficient of $i^{\text {th }}$ genotype at $j^{\text {th }}$

\section{Results and Discussion}

Bartlett's test indicated homogenous error variance for the traits in each of eight environments and allowed to proceed further for pooled analysis across environments. Genotype, environment 
variances and genotype $\times$ environment interaction plant $^{-1}$ for genotypes Table (3). were significant for all traits except number of pods

Table 3. Combined analysis of variance of evaluated genotypes over different environments.

\begin{tabular}{|c|c|c|c|c|c|c|c|c|c|}
\hline $\begin{array}{l}\text { Source of } \\
\text { Variance }\end{array}$ & df & $\begin{array}{c}\text { Pod } \\
\text { weight } \\
\text { plant }^{-1}\end{array}$ & $\begin{array}{c}\text { No. of } \\
\text { pods } \\
\text { plant }^{-1}\end{array}$ & $\begin{array}{l}\text { No. of } \\
\text { seeds } \\
\text { plant }^{-1}\end{array}$ & $\begin{array}{c}\text { Seed } \\
\text { weight } \\
\text { plant }(g)\end{array}$ & $\begin{array}{c}\text { Shelling } \\
\%\end{array}$ & $\begin{array}{l}\text { 100- pod } \\
\text { weight } \\
\text { (g) }\end{array}$ & $\begin{array}{c}100- \\
\text { seed } \\
\text { weight }\end{array}$ & $\begin{array}{c}\text { Pod } \\
\text { yield } \\
\text { fed }^{-1} \\
\text { (ardab) }\end{array}$ \\
\hline Genotypes(G) & 7 & $\underset{* * *}{3790.91}$ & 112.67 & $\underset{*}{1779.13}$ & 1883.01* $_{*}^{*}$ & $16.97^{* *}$ & $\underset{*}{\text { 8342.00* }}$ & 617.66 $_{*}^{*}$ & $\underset{*}{121.58}$ \\
\hline $\begin{array}{l}\text { Environments( } \\
\text { E) }\end{array}$ & 7 & $\underset{* *}{3878.68}$ & $\underset{*}{714.19}$ & 839.00** & ${ }_{*}^{1611.21} 1^{*}$ & $4.46^{*}$ & $\underset{* *}{2979.43}$ & $\underset{* *}{431.88}$ & $\underset{*}{247.88}$ \\
\hline $\mathbf{G} \times \mathbf{E}$ & 49 & $\underset{*}{390.35^{*}}$ & $100.91^{*}$ & 203.099* & $167.13^{* *}$ & $3.45^{*}$ & $668.24^{* *}$ & $37.76^{*}$ & $4.37^{*}$ \\
\hline Pooled error & 128 & 62.78 & 23.57 & 41.82 & 26.66 & 1.39 & 157.19 & 8.49 & 3.11 \\
\hline Total & 191 & & & & & & & & \\
\hline
\end{tabular}

The existence of significant difference among the genotypes was the representation of the difference of genetic potentiality of the genotypes for the evaluated characteristics; also, the existence of significant difference among the studied environments represents the significant variety effect in the additive structure of data for the evaluated characteristics among the environments. Similar results were reported by Minimol et al. (2001), Mahasi et al. (2006) and Zerihun et al. (2011).

Mean performance of genotypes for eight studied traits is shown in Table (4). Results revealed that the means values varied from 83.60 to $123.35 \mathrm{~g}$ with an average of $97.88 \mathrm{~g}$ for pod weight plant ${ }^{-1}$, from 43.78 to 50.19 with an average of 46.5 for number of pods plant $^{-1}$, from 65.52 to 83.91 with an average of 73.67 for number of seeds plant ${ }^{-1}$, from 53.28 to 81.81 with an average of $63.62 \mathrm{~g}$ for seed weight plant $^{-1}$, from 63.63 to 66.42 with an average of 64.93 for shelling percentage $\%$, from 186.98 to 245.36 with an average of $202.47 \mathrm{~g}$ for 100 - pod weight, from 81.51 to 97.27 with an average of $86.13 \mathrm{~g}$ for 100 -seed weight, and from 21.44 to 27.64 with an average of 25.61 ardab for pod yield $\mathrm{fed}^{-1}$. The genotype Sohag 120 produced the highest values for all studied traits. Regarding the environments, (Table 4), there were significant effects on the studied traits, indicating a wide range of environmental effects. Assuit environment (4) had the highest mean values of environments for pod weight plant ${ }^{-1}$, number of seeds plant $^{-1}$, Seed weight plant ${ }^{-1}(\mathrm{~g})$ and100- pod weight, and Assuit environment (3) had the highest mean values of environments for pod yield fed. ${ }^{-1}$, Meanwhile, Shandweel environment (6) had the highest mean values of environments for 100- seed weight. The reverse trend was true for different traits and environments. In this connection, some investigators emphasized that environments had great effects on peanut genotypes traits Therefore, Assuit environments were the best environment. Similar results were reported by (Abd El-Rahman et al. (2016) and Minde et al. (2017).
The mean squares due to genotype were highly significant for all the studied characters except number of pods plant $^{-1}$ (Table 5), which revealed the presence of substantial amount of variation among the groundnut genotypes. The significant mean squares for environment (linear) for various traits were also reported by Habib et al. (1986) and Patil et al. (2014). Variance due to genotypes $\times$ environment (linear) was significant for pod weight plant $^{-1}$, No. of seeds plant ${ }^{-1}$, seed weight plant (g) and pod yield $\mathrm{fed}^{-1}$ (ardeb). Significance of variance due to environment (linear) was observed for all the characters studied except shelling percentage and 100-pod weight (g), (Table 5). The higher magnitude of mean squares for environment (linear) compared to genotypes $x$ environments (linear) indicated that linear response of environment accounted for the major part of total variation for all studied characters and may be responsible for high adaptation in relation to yield and other traits. Therefore, prediction of performance of genotypes over environments would be possible for the various characters. Similar findings were reported by Thaware (2009), Pradhan et al. (2010), Habib et al. (1986) and Patil et al. (2014). Variance due to pooled deviation was significant for all studied characters indicating that genotypes differed considerably with respect to their stability. The significant pooled deviation (Non-linear) for various traits were also reported by Senapati et al. (2004), Chuni Lal et al. (2006) and Patil et al. (2014). Interactions of genotypes with environments obtained as the environment + genotype $\times$ environments $(e+g \times e)$ were significant for all characters (Table 5), which suggested the distinct nature of environments and genotype $\times$ environment interactions in phenotypic expression. The significant environment + (genotype $\times$ environment $)$ interactions for various traits were also reported by Joshi $\boldsymbol{e t}$ al. (2003) and Patil et al. (2014). 
Table 4. Mean performance of studied traits over different environments.

\begin{tabular}{|c|c|c|c|c|c|c|c|c|c|c|}
\hline \multirow{3}{*}{ Trait } & \multirow{3}{*}{$\begin{array}{l}\text { Env. code } \\
\text { Genotypes }\end{array}$} & \multicolumn{4}{|c|}{ Assuit } & \multicolumn{4}{|c|}{ Shandweel } & \multirow{3}{*}{ Mean } \\
\hline & & 2014 & 2015 & 2016 & 2017 & 2014 & 2015 & 2016 & 2017 & \\
\hline & & Env.1 & Env.2 & Env.3 & Env.4 & Env.5 & Env.6 & Env.7 & Env.8 & \\
\hline \multirow{9}{*}{ 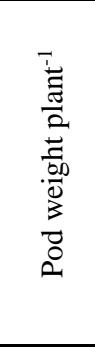 } & Giza 6 & 81.190 & 94.30 & 100.16 & 101.96 & 79.250 & 99.350 & 80.92 & 92.12 & 91.16de \\
\hline & SOHAG112 & 85.82 & 87.44 & 92.163 & 90.00 & 78.40 & 103.02 & 77.907 & 85.69 & $87.56 \mathrm{ef}$ \\
\hline & LINE9 & 84.78 & 97.70 & 94.17 & 105.89 & 67.87 & 75.50 & 69.55 & 73.33 & 83.60f \\
\hline & INTRO. 508 & 90.63 & 88.93 & 97.94 & 123.27 & 84.53 & 92.10 & 78.54 & 92.01 & $93.49 \mathrm{~cd}$ \\
\hline & VAC-R92 & 98.78 & 75.18 & 91.33 & 146.08 & 93.90 & 110.39 & 89.99 & 107.10 & 101.59b \\
\hline & SOHAG116 & 101.75 & 92.34 & 133.67 & 137.27 & 72.93 & 118.24 & 75.88 & 115.96 & 106.01b \\
\hline & SOHAG119 & 102.86 & 78.93 & 105.02 & 116.02 & 74.57 & 101.63 & 82.77 & 108.60 & $96.29 \mathrm{c}$ \\
\hline & SOHAG120 & 147.10 & 93.58 & 110.17 & 144.21 & 113.75 & 133.76 & 106.45 & 137.73 & 123.35a \\
\hline & Mean & $99.12 \mathrm{c}$ & $88.55 d$ & 103.08bc & $120.59 a$ & 83.15e & $104.25 b$ & $82.75 e$ & 101.57bc & 97.88 \\
\hline \multirow{9}{*}{ 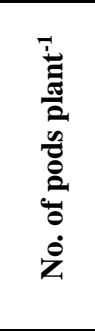 } & Giza 6 & 46.41 & 52.67 & 53.47 & 49.95 & 36.89 & 44.47 & 35.62 & 44.55 & $45.50 \mathrm{bc}$ \\
\hline & SOHAG112 & 42.92 & 43.73 & 59.50 & 41.59 & 45.66 & 51.83 & 43.37 & 49.10 & 47.22b \\
\hline & LINE9 & 40.24 & 58.00 & 51.55 & 46.13 & 36.67 & 39.77 & 34.85 & 43.29 & 43.81c \\
\hline & INTRO. 508 & 52.22 & 46.39 & 56.07 & 56.22 & 36.92 & 39.17 & 36.79 & 44.83 & 46.07bc \\
\hline & VAC-R92 & 46.53 & 34.46 & 46.93 & 62.78 & 45.12 & 49.20 & 43.34 & 52.81 & 47.65ab \\
\hline & SOHAG116 & 42.80 & 37.73 & 63.74 & 58.08 & 35.92 & 52.72 & 36.02 & 55.22 & 47.78ab \\
\hline & SOHAG119 & 46.84 & 39.53 & 51.09 & 50.19 & 33.68 & 43.91 & 37.89 & 47.07 & 43.78c \\
\hline & SOHAG120 & 60.87 & 42.00 & 49.19 & 55.16 & 43.41 & 51.47 & 45.51 & 53.89 & 50.19a \\
\hline & Mean & $47.35 c$ & $44.32 d$ & 53.94b & 52.52b & $39.28 \mathrm{e}$ & $46.57 \mathrm{~cd}$ & $39.18 \mathrm{e}$ & $48.85 a$ & 46.5 \\
\hline \multirow{9}{*}{ 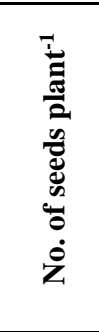 } & Giza 6 & 67.16 & 76.27 & 86.47 & 80.08 & 61.37 & 66.51 & 62.64 & 70.89 & $71.43 c$ \\
\hline & SOHAG112 & 71.74 & 69.85 & 77.69 & 66.02 & 64.90 & 67.41 & 65.21 & 71.81 & $69.33 \mathrm{c}$ \\
\hline & LINE9 & 66.33 & 81.19 & 78.44 & 77.97 & 54.38 & 52.74 & 56.44 & 56.65 & $65.52 d$ \\
\hline & INTRO. 508 & 74.33 & 73.69 & 80.77 & 86.40 & 63.93 & 63.10 & 64.17 & 72.21 & $72.33 c$ \\
\hline & VAC-R92 & 75.73 & 60.08 & 77.20 & 99.37 & 68.95 & 79.57 & 69.15 & 79.50 & 76.19b \\
\hline & SOHAG116 & 75.02 & 68.88 & 109.48 & 96.15 & 55.04 & 86.88 & 56.72 & 88.21 & $79.55 b$ \\
\hline & SOHAG119 & 71.55 & 65.97 & 86.75 & 82.02 & 52.30 & 73.38 & 58.79 & 77.69 & $71.06 \mathrm{c}$ \\
\hline & SOHAG120 & 93.32 & 66.96 & 80.06 & 95.93 & 76.44 & 90.57 & 73.58 & 94.42 & 83.91a \\
\hline & Mean & $74.40 \mathrm{bc}$ & 70.36d & $84.61 a$ & $85.49 a$ & $62.16 \mathrm{e}$ & $72.52 \mathrm{~cd}$ & $63.34 \mathrm{e}$ & $76.43 \mathrm{~b}$ & 73.67 \\
\hline \multirow{9}{*}{ 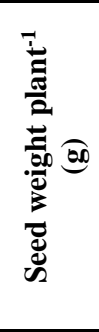 } & Giza 6 & 53.09 & 62.51 & 67.32 & 67.09 & 51.79 & 63.27 & 53.33 & 60.48 & 59.86d \\
\hline & SOHAG112 & 56.43 & 57.10 & 59.05 & 58.64 & 50.59 & 65.51 & 49.44 & 54.38 & $56.39 \mathrm{e}$ \\
\hline & LINE9 & 53.78 & 64.04 & 60.89 & 68.07 & 42.89 & 46.01 & 43.61 & 46.97 & $53.28 f$ \\
\hline & INTRO. 508 & 59.77 & 58.09 & 62.89 & 77.71 & 54.34 & 60.32 & 51.26 & 60.27 & $60.58 d$ \\
\hline & VAC-R92 & 63.26 & 48.83 & 59.69 & 94.48 & 59.16 & 73.79 & 57.69 & 68.01 & $65.62 c$ \\
\hline & SOHAG116 & 68.22 & 59.89 & 88.11 & 88.25 & 46.78 & 78.67 & 48.97 & 74.02 & $69.11 b$ \\
\hline & SOHAG119 & 64.95 & 52.12 & 68.53 & 74.30 & 47.47 & 65.32 & 54.47 & 71.17 & $62.29 d$ \\
\hline & SOHAG120 & 94.76 & 62.75 & 73.59 & 94.75 & 75.68 & 89.09 & 71.36 & 92.50 & 81.81a \\
\hline & Mean & $64.29 \mathrm{c}$ & 58.17d & $67.51 b$ & 77.91a & $53.59 \mathrm{e}$ & $67.75 b$ & $53.77 \mathrm{e}$ & 65.97bc & 63.62 \\
\hline \multirow{9}{*}{ 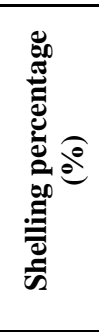 } & G1 & 65.45 & 66.27 & 67.21 & 65.79 & 65.34 & 63.69 & 65.92 & 65.69 & $65.67 \mathrm{~b}$ \\
\hline & SOHAG112 & 65.75 & 65.30 & 64.08 & 65.19 & 64.53 & 63.63 & 63.48 & 63.44 & $64.43 d$ \\
\hline & LINE9 & 63.37 & 65.66 & 64.71 & 64.29 & 63.24 & 60.94 & 62.71 & 64.09 & $63.63 \mathrm{e}$ \\
\hline & INTRODUCE5 & 65.74 & 65.35 & 64.27 & 63.02 & 64.31 & 65.45 & 65.27 & 65.49 & $64.87 \mathrm{~cd}$ \\
\hline & VAC-R92 & 63.93 & 64.94 & 65.38 & 64.71 & 63.01 & 66.86 & 64.10 & 63.51 & $64.56 \mathrm{~cd}$ \\
\hline & SOHAG116 & 67.06 & 64.68 & 65.99 & 64.32 & 64.11 & 66.52 & 64.55 & 63.81 & 65.13bc \\
\hline & SOHAG119 & 63.38 & 66.00 & 65.28 & 63.99 & 63.67 & 64.26 & 65.82 & 65.53 & $64.74 \mathrm{~cd}$ \\
\hline & SOHAG120 & 64.43 & 67.04 & 66.77 & 65.70 & 66.55 & 66.63 & 67.05 & 67.19 & $66.42 \mathrm{a}$ \\
\hline & Mean & $64.89 \mathrm{bc}$ & $65.66 \mathrm{a}$ & $65.46 \mathrm{ab}$ & $64.63 c$ & $64.35 \mathrm{c}$ & $64.75 \mathrm{c}$ & $64.86 \mathrm{bc}$ & $64.85 b c$ & 64.93 \\
\hline \multirow{9}{*}{ 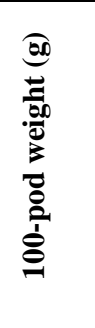 } & Giza 6 & 175.13 & 181.05 & 188.19 & 204.30 & 214.91 & 223.47 & 227.27 & 206.83 & 202.64d \\
\hline & SOHAG112 & 200.03 & 199.97 & 154.91 & 216.30 & 171.74 & 198.72 & 179.63 & 174.49 & $186.98 \mathrm{e}$ \\
\hline & LINE9 & 210.80 & 171.50 & 183.28 & 229.67 & 185.02 & 189.91 & 199.77 & 169.38 & $192.42 \mathrm{e}$ \\
\hline & INTRO. 508 & 173.91 & 191.61 & 177.47 & 219.15 & 229.20 & 234.89 & 213.76 & 205.39 & $205.67 d$ \\
\hline & VAC-R92 & 212.23 & 221.54 & 195.73 & 233.23 & 208.50 & 224.51 & 207.84 & 202.66 & $213.28 c$ \\
\hline & SOHAG116 & 237.56 & 250.30 & 210.86 & 236.16 & 203.26 & 224.90 & 210.85 & 209.95 & $222.98 b$ \\
\hline & SOHAG119 & 219.13 & 201.97 & 206.68 & 231.07 & 221.41 & 231.67 & 218.37 & 230.55 & 220.11bc \\
\hline & SOHAG120 & 241.58 & 223.57 & 224.35 & 261.15 & 262.70 & 260.13 & 233.89 & 255.54 & 245.36a \\
\hline & Mean & $208.79 b$ & 205.19b & $192.69 c$ & 228.88a & 212.09b & 223.52a & 211.42b & $206.85 b$ & 211.18 \\
\hline
\end{tabular}


Table 4. Continued.

\begin{tabular}{|c|c|c|c|c|c|c|c|c|c|c|}
\hline \multirow[b]{2}{*}{ Trait } & \multirow[b]{2}{*}{$\begin{array}{l}\text { Env. code } \\
\text { Genotypes }\end{array}$} & \multicolumn{4}{|c|}{ Assuit } & \multicolumn{4}{|c|}{ Shandweel } & \multirow[b]{2}{*}{ Mean } \\
\hline & & $\begin{array}{l}2014 \\
\text { Env.1 }\end{array}$ & $\begin{array}{l}2015 \\
\text { Env.2 }\end{array}$ & $\begin{array}{l}2016 \\
\text { Env.3 }\end{array}$ & $\begin{array}{l}2017 \\
\text { Env.4 }\end{array}$ & $\begin{array}{l}2014 \\
\text { Env.5 }\end{array}$ & $\begin{array}{l}2015 \\
\text { Env.6 }\end{array}$ & $\begin{array}{l}2016 \\
\text { Env.7 }\end{array}$ & $\begin{array}{l}2017 \\
\text { Env.8 }\end{array}$ & \\
\hline \multirow{9}{*}{ 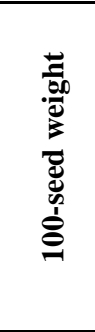 } & Giza 6 & 79.15 & 81.98 & 78.17 & 83.88 & 84.48 & 95.31 & 85.27 & 85.33 & 84.19de \\
\hline & SOHAG112 & 78.64 & 81.91 & 76.05 & 88.79 & 78.00 & 97.14 & 75.82 & 75.73 & 81.51f \\
\hline & LINE9 & 81.34 & 78.74 & 77.63 & 87.28 & 78.92 & 87.37 & 77.29 & 83.01 & $81.45 f$ \\
\hline & INTRO. 508 & 80.42 & 78.85 & 77.86 & 90.09 & 85.02 & 96.03 & 79.88 & 83.49 & $83.96 \mathbf{e}$ \\
\hline & VAC-R92 & 83.60 & 81.35 & 77.68 & 95.09 & 85.85 & 92.77 & 83.44 & 85.50 & $85.66 \mathrm{~cd}$ \\
\hline & SOHAG116 & 91.06 & 86.86 & 80.44 & 91.94 & 85.12 & 90.54 & 86.37 & 83.99 & 87.04bc \\
\hline & SOHAG119 & 90.80 & 79.06 & 79.06 & 90.50 & 90.79 & 89.02 & 92.64 & 91.59 & 87.93b \\
\hline & SOHAG120 & 101.50 & 93.67 & 91.94 & 98.69 & 99.01 & 98.34 & 97.03 & 97.97 & 97.27a \\
\hline & Mean & $85.81 c$ & 82.81d & $79.86 \mathrm{e}$ & $90.79 b$ & $85.90 c$ & 93.32a & $84.72 c$ & $85.83 c$ & 86.13 \\
\hline \multirow{9}{*}{ 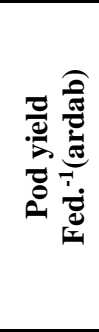 } & Giza 6 & 22.91 & 23.55 & 28.76 & 25.11 & 23.16 & 20.32 & 20.91 & 19.69 & 23.05de \\
\hline & SOHAG112 & 23.35 & 23.22 & 28.50 & 23.94 & 19.51 & 18.04 & 18.86 & 17.75 & $21.65 f$ \\
\hline & LINE9 & 24.02 & 23.58 & 28.21 & 22.88 & 19.67 & 17.54 & 17.83 & 17.75 & 21.44f \\
\hline & INTRO. 508 & 24.05 & 23.11 & 28.92 & 24.36 & 23.71 & 17.67 & 17.93 & 17.00 & 22.09ef \\
\hline & VAC-R92 & 21.77 & 23.19 & 28.45 & 26.66 & 25.49 & 22.26 & 21.06 & 20.37 & $23.66 \mathrm{~cd}$ \\
\hline & SOHAG116 & 26.82 & 24.54 & 31.30 & 26.61 & 22.96 & 23.06 & 21.53 & 20.44 & $24.66 c$ \\
\hline & SOHAG119 & 29.87 & 27.31 & 31.29 & 26.43 & 25.44 & 24.49 & 23.35 & 22.18 & $26.29 \mathrm{~b}$ \\
\hline & SOHAG120 & 27.98 & 27.57 & 33.29 & 28.66 & 27.47 & 26.48 & 25.84 & 23.86 & $27.64 \mathrm{a}$ \\
\hline & Mean & 25.09 bc & 24.51c & $29.84 a$ & $25.58 b$ & 23.43d & $21.23 \mathrm{e}$ & $20.91 \mathrm{e}$ & $19.88 f$ & 23.81 \\
\hline
\end{tabular}

Table 5. Analysis of variance for pod yield and yield contributing traits under different environments

\begin{tabular}{|c|c|c|c|c|c|c|c|c|c|}
\hline SOV & df & $\begin{array}{c}\text { Pod } \\
\text { weight } \\
\text { plant }^{-1}\end{array}$ & $\begin{array}{l}\text { No. of } \\
\text { pods } \\
\text { plant }^{-1}\end{array}$ & $\begin{array}{l}\text { No. of } \\
\text { seeds } \\
\text { plant }^{-1}\end{array}$ & $\begin{array}{c}\text { Seed } \\
\text { weight } \\
\text { plant (g) }\end{array}$ & $\begin{array}{c}\text { Shelling } \\
\%\end{array}$ & $\begin{array}{c}\text { 100- pod } \\
\text { weight }(\mathrm{g})\end{array}$ & $\begin{array}{c}\text { 100- seed } \\
\text { weight }\end{array}$ & $\begin{array}{c}\text { Pod } \\
\text { yield } \\
\text { fed }^{-1} \\
\text { (ardeb) }\end{array}$ \\
\hline Genotypes & 7 & $1263.67 * *$ & 37.58 & $279.67 * *$ & $627.66 * *$ & $5.65 * *$ & $2780.71 * *$ & $205.88 * *$ & $0.67 * *$ \\
\hline $\begin{array}{l}\text { Env. + } \\
\text { (Genotypes x } \\
\text { Env.) }\end{array}$ & 56 & $275.46 * *$ & $59.19 * *$ & $133.37 * *$ & $115.88 * *$ & 1.26 & 319.05* & $29.00 * *$ & $0.19 * *$ \\
\hline $\begin{array}{l}\text { Environment } \\
\text { (linear) }\end{array}$ & 1 & $9050.49 * *$ & $1666.57 * *$ & 4151.31** & $3759.39 * *$ & 0.19 & 6952.14 & $1007.69 * *$ & $9.57 * *$ \\
\hline $\begin{array}{l}\text { Genotype } x \\
\text { Environment } \\
\text { (linear) }\end{array}$ & 7 & $234.85 * *$ & 38.211 & $142.22 * *$ & $100.84 * *$ & 0.48 & 111.66 & 17.29 & $0.04 * *$ \\
\hline $\begin{array}{l}\text { Pooled } \\
\text { deviation }\end{array}$ & 48 & $98.57 * *$ & $28.77 * *$ & $48.37 * *$ & $42.17 * *$ & $1.11 * *$ & 211.1** & $10.32 * *$ & $0.019 * *$ \\
\hline Giza6 & 6 & 43.93 & $18.43 * *$ & 23.46 & 20.08* & 0.75 & $333.26 * *$ & $12.05 * *$ & 0.007 \\
\hline Sohag112 & 6 & 44.99 & $33.58 * *$ & 14.54 & 16.35 & $0.93 * *$ & $197.90 * *$ & $20.87 * *$ & 0.013 \\
\hline Line9 & 6 & $146.49 * *$ & $52.43 * *$ & $104.51 * *$ & $74.15 * *$ & $1.49 * *$ & 300.27 & 3.89 & 0.019 \\
\hline Intr.508 & 6 & 34.71 & $18.04 * *$ & 18.78 & 9.51 & 0.93 & $282.46 * *$ & 4.59 & 0.019 \\
\hline VAC-R92 & 6 & $154.38 * *$ & $51.78 * *$ & $73.64 * *$ & $66.78 * *$ & $1.49 * *$ & 52.03 & 3.26 & $0.047 *$ \\
\hline Sohag116 & 6 & $90.34 * *$ & $20.44 * *$ & $45.21 * *$ & $36.64 * *$ & $1.64 * *$ & $312.10 * *$ & $7.27 * *$ & 0.012 \\
\hline Sohag119 & 6 & $\mathbf{3 7 . 4 7}$ & 3.09 & 10.35 & $17.32 *$ & 0.69 & 64.84 & $24.09 * *$ & 0.025 \\
\hline Sohag120 & 6 & $236.22 * *$ & $32.31 * *$ & $96.48 * *$ & $96.51 * *$ & $0.94 * *$ & $145.95 * *$ & $6.53 * *$ & 0.009 \\
\hline Pooled error & 128 & 22.74 & 7.8 & 14.06 & 9.63 & 0.46 & $50.14 * *$ & 2.89 & 0.017 \\
\hline
\end{tabular}

In the present investigation, model proposed by Eberhart and Rusell (1966) was used for analysis of $\mathrm{G} \times \mathrm{E}$ interactions. This model considered both linear $(b i)$ and non-linear $\left(S^{2} d i\right)$ components of $\mathrm{G} \times \mathrm{E}$ interactions for the prediction of performance of the individual genotype. Higher mean performance of genotype for various characters along with regression coefficient (bi) as measures of responsive and deviation from regression $\left(S^{2} d i\right)$ as a measure of stability were used to assess the stability and suitability of performance over environments. The high mean performance of genotypes was taken on the basis of average performance of all genotype as population mean.
The bi value was approached near unity in peanut genotypes VAC-R92, Sohag116, Sohag119 and Sohag120 for pod weight and seed weight, genotypes 6 and 7 for number of pods and number of seedsplant ${ }^{-1}$ genotypes $1,3,5$ and 7 for shelling percentage , genotypes $2,3,4$ and 8 for 100-pod weight, genotypes 2,4 and 5 for 100- seed weight genotypes 2, 3, 4 and 6 for pod yield $\mathrm{fed}^{-1}$., where the value of bi almost approached unity, indicating average response to the fluctuating environmental conditions prevailed.

Genotypes 5, 6 and 8 had the highest pod weight plant $^{-1}$, number of pods plant $^{-1}$, number of seeds plant $^{-1}$ and seed weight plant $^{-1}$ among the tested 
genotypes, as they had higher means of pods plant ${ }^{-1}$ than overall mean value of (46.5). Peanut genotypes 5, 7 and 8 for 100- pod weight (g), genotypes 6 and 7 for 100 - seed weight $(\mathrm{g})$, genotypes 6,7 and 8 for pod yield $\mathrm{fed}^{-1}$ (ard.). These genotypes are suitable especially for favorable growing seasons as they had high (bi) value $(b>1)$. These results were in accordance with the Pradhan et al. (2010) and Patil et al. (2014).
Peanut genotype Sohag112 recorded the highest number of pods plant ${ }^{-1}$ over the grand mean (46.50), whereas genotypes 6 and 8 gave highest shelling percentage, genotype Sohag 116 gave the highest 100- pod weight and Sohag 120 gave the highest number of seedsplant ${ }^{-1}$ indicating that these genotypes are fitted, for less favorable locations as they had low (bi) value $(b<1)$ these results agree with those reported by Abd El-Rahman et al. (2016) and Hasan et al. (2018)

Table 6. Estimates of stability parameters for eight peanut genotypes in all studied characters.

\begin{tabular}{|c|c|c|c|c|c|c|c|c|c|c|c|c|}
\hline \multirow[t]{2}{*}{ Genotypes } & \multicolumn{3}{|c|}{ Pod weight plant $^{-1}$} & \multicolumn{3}{|c|}{ No. of pods plant ${ }^{-1}$} & \multicolumn{3}{|c|}{ No. of seeds plant ${ }^{-1}$} & \multicolumn{3}{|c|}{$\begin{array}{c}\text { Seed weight } \text { plant }^{-1} \\
\text { (g) }\end{array}$} \\
\hline & $\overline{\bar{x}}$ & $\mathbf{B}_{\mathbf{i}}$ & $\mathbf{S}_{\mathbf{d}}^{2}$ & $\bar{x}$ & $\mathbf{B}_{\mathbf{i}}$ & $\mathbf{S}_{\mathbf{d}}^{2}$ & $\bar{x}$ & $\mathbf{B}_{\mathbf{i}}$ & $\mathbf{S}_{\mathbf{d}}^{2}$ & $\bar{x}$ & $\mathbf{B}_{\mathbf{i}}$ & $\mathbf{S}_{\mathrm{d}}^{2}$ \\
\hline Giza 6 & 91.16 & $0.56^{*}$ & 621.75 & 45.50 & $\begin{array}{c}0.98 * \\
*\end{array}$ & 309.26 & $\begin{array}{c}71.4 \\
3\end{array}$ & $\begin{array}{c}0.88^{*} \\
*\end{array}$ & 545.61 & $\begin{array}{c}59.8 \\
6\end{array}$ & $0.58 *$ & $\begin{array}{c}280.5 \\
2\end{array}$ \\
\hline $\begin{array}{l}\text { SOHAG11 } \\
2\end{array}$ & 87.56 & 0.39 & 449.73 & 47.22 & 0.51 & 255.09 & $\begin{array}{c}69.3 \\
3\end{array}$ & 0.29 & 133.46 & $\begin{array}{c}56.3 \\
9\end{array}$ & 0.42 & $\begin{array}{c}181.7 \\
4\end{array}$ \\
\hline Line9 & 83.60 & 0.69 & $\begin{array}{c}1424.9 \\
2\end{array}$ & 43.81 & 0.74 & 427.23 & $\begin{array}{c}65.5 \\
2\end{array}$ & 0.87 & $\begin{array}{c}1016.4 \\
5\end{array}$ & $\begin{array}{c}53.2 \\
9\end{array}$ & 0.72 & $\begin{array}{c}686.9 \\
3\end{array}$ \\
\hline Intro.,508 & 93.49 & $\begin{array}{l}0.96^{*} \\
*\end{array}$ & $\begin{array}{c}1243.5 \\
1\end{array}$ & 46.07 & $\begin{array}{c}1.29 * \\
*\end{array}$ & 459.77 & $\begin{array}{c}72.3 \\
3\end{array}$ & $\begin{array}{c}0.86^{*} \\
*\end{array}$ & 497.36 & $\begin{array}{c}60.5 \\
8\end{array}$ & $\begin{array}{l}0.89 * \\
*\end{array}$ & $\begin{array}{c}431.6 \\
9\end{array}$ \\
\hline VAC-R92 & $\begin{array}{c}101.5 \\
9\end{array}$ & $\begin{array}{c}1.38 * \\
*\end{array}$ & $\begin{array}{c}3091.4 \\
3\end{array}$ & 47.65 & 0.84 & 458.75 & $\begin{array}{c}76.1 \\
9\end{array}$ & $0.96^{*}$ & 922.40 & $\begin{array}{c}65.6 \\
2\end{array}$ & $\begin{array}{c}1.41 * \\
*\end{array}$ & $\begin{array}{c}661.8 \\
4\end{array}$ \\
\hline Sohag116 & $\begin{array}{c}106.0 \\
1\end{array}$ & $\begin{array}{c}1.79 * \\
*\end{array}$ & $\begin{array}{c}4197.8 \\
3\end{array}$ & 47.78 & $\begin{array}{c}1.86^{*} \\
*\end{array}$ & 845.43 & $\begin{array}{c}79.5 \\
5\end{array}$ & $\begin{array}{c}2.09 * \\
*\end{array}$ & $\begin{array}{l}2556.4 \\
9\end{array}$ & $\begin{array}{c}69.1 \\
1\end{array}$ & $\begin{array}{c}1.85^{*} \\
*\end{array}$ & $\begin{array}{c}870.6 \\
8\end{array}$ \\
\hline Sohag119 & 96.29 & $\begin{array}{c}1.12 * \\
*\end{array}$ & $\begin{array}{c}1644.5 \\
8\end{array}$ & 43.78 & $\begin{array}{c}1.09 * \\
*\end{array}$ & 269.32 & $\begin{array}{c}71.0 \\
6\end{array}$ & $\begin{array}{c}1.30^{*} \\
*\end{array}$ & 944.26 & $\begin{array}{c}62.2 \\
9\end{array}$ & $\begin{array}{c}1.09 * \\
*\end{array}$ & $\begin{array}{c}512.3 \\
2\end{array}$ \\
\hline Sohag120 & $\begin{array}{c}123.3 \\
5 \\
\end{array}$ & 1.09 & $\begin{array}{c}2752.1 \\
2 \\
\end{array}$ & 50.19 & 0.68 & 289.93 & $\begin{array}{c}83.9 \\
1\end{array}$ & 0.73 & 852.57 & $\begin{array}{c}81.8 \\
1\end{array}$ & 1.03 & $\begin{array}{c}485.2 \\
5\end{array}$ \\
\hline Mean & 97.88 & 1 & & 46.50 & 1 & & 73.66 & 1 & & 63.62 & 1 & \\
\hline \multirow[t]{3}{*}{ SE } & 3.75 & 0.29 & & 2.03 & 0.37 & & 2.63 & 0.31 & & 2.45 & 0.29 & \\
\hline & \multicolumn{3}{|c|}{ Shelling \% } & \multicolumn{3}{|c|}{ 100- pod weight (g) } & \multicolumn{3}{|c|}{ 100- seed weight } & \multicolumn{3}{|c|}{ Pod yield fed $^{-1}$ (ard.) } \\
\hline & $\overline{\boldsymbol{x}}$ & $\mathbf{B}_{\mathbf{i}}$ & $\mathbf{S}_{\mathbf{d}}^{2}$ & $\overline{\boldsymbol{x}}$ & $\mathbf{B}_{\mathbf{i}}$ & $\mathbf{S}_{\mathbf{d}}^{2}$ & $\bar{x}$ & $\mathbf{B}_{\mathbf{i}}$ & $\mathbf{S}_{\mathbf{d}}^{2}$ & $\overline{\bar{x}}$ & $\mathbf{B}_{\mathbf{i}}$ & $\mathbf{S}_{\mathrm{d}}^{2}$ \\
\hline Giza 6 & 65.67 & 1.37 & 6.89 & $\begin{array}{c}202.6 \\
4\end{array}$ & 0.86 & $\begin{array}{c}2643.0 \\
5\end{array}$ & $\begin{array}{c}84.1 \\
9\end{array}$ & $\begin{array}{c}0.98 * \\
*\end{array}$ & 192.96 & $\begin{array}{c}23.0 \\
5\end{array}$ & $\begin{array}{c}0.89 * \\
*\end{array}$ & 60.51 \\
\hline Sohag112 & 64.43 & 0.32 & 5.72 & $\begin{array}{c}186.9 \\
8\end{array}$ & $1.37 *$ & $\begin{array}{c}2807.5 \\
6\end{array}$ & $\begin{array}{c}81.5 \\
1\end{array}$ & $\begin{array}{c}1.51 * \\
*\end{array}$ & 413.55 & $\begin{array}{c}21.6 \\
4\end{array}$ & $\begin{array}{c}1.14^{*} \\
*\end{array}$ & 98.11 \\
\hline Line 9 & 63.63 & 2.03 & 14.27 & $\begin{array}{c}192.4 \\
2\end{array}$ & 1.12 & $\begin{array}{c}2892.3 \\
8\end{array}$ & $\begin{array}{c}81.4 \\
5\end{array}$ & $\begin{array}{c}0.86^{*} \\
*\end{array}$ & 117.08 & $\begin{array}{c}21.4 \\
3\end{array}$ & $\begin{array}{c}1.16^{*} \\
*\end{array}$ & $\begin{array}{c}104.2 \\
0\end{array}$ \\
\hline Intro.,508 & 64.87 & 0.54 & 5.95 & $\begin{array}{c}205.6 \\
7\end{array}$ & $1.50 *$ & $\begin{array}{c}3656.0 \\
3\end{array}$ & $\begin{array}{c}83.9 \\
6\end{array}$ & $\begin{array}{c}1.41 * \\
*\end{array}$ & 277.04 & $\begin{array}{c}22.0 \\
9\end{array}$ & 1.26 & $\begin{array}{c}122.0 \\
1\end{array}$ \\
\hline VAC-R92 & 64.56 & 1.00 & 10.22 & $\begin{array}{c}213.2 \\
8\end{array}$ & $\begin{array}{c}0.93 * \\
*\end{array}$ & $\begin{array}{c}1066.1 \\
7\end{array}$ & $\begin{array}{c}85.6 \\
6\end{array}$ & $\begin{array}{c}1.29 * \\
*\end{array}$ & 231.02 & $\begin{array}{c}23.6 \\
6\end{array}$ & $\begin{array}{c}0.76^{*} \\
*\end{array}$ & 58.72 \\
\hline Sohag116 & 65.13 & 0.61 & 10.35 & $\begin{array}{c}222.9 \\
8\end{array}$ & 0.36 & $\begin{array}{c}1989.1 \\
3\end{array}$ & $\begin{array}{c}87.0 \\
4\end{array}$ & $\begin{array}{c}0.72 * \\
*\end{array}$ & 109.32 & $\begin{array}{c}24.6 \\
6\end{array}$ & $\begin{array}{c}1.06^{*} \\
*\end{array}$ & 85.64 \\
\hline Sohag119 & 64.74 & 1.60 & 7.45 & $\begin{array}{c}220.1 \\
0\end{array}$ & $0.75 *$ & 877.97 & $\begin{array}{c}87.9 \\
3\end{array}$ & 0.76 & 217.22 & $\begin{array}{c}26.2 \\
9\end{array}$ & $\begin{array}{l}0.91 * \\
*\end{array}$ & 68.48 \\
\hline Sohag120 & 66.42 & 0.55 & 6.02 & $\begin{array}{c}245.3 \\
6 \\
\end{array}$ & $1.10^{*}$ & $\begin{array}{c}1934.4 \\
0 \\
\end{array}$ & $\begin{array}{c}97.2 \\
7 \\
\end{array}$ & 0.46 & 65.99 & $\begin{array}{c}27.6 \\
4 \\
\end{array}$ & $\begin{array}{c}0.82 * \\
* \\
\end{array}$ & 52.05 \\
\hline Mean & 64.93 & 1 & & $\begin{array}{c}211.1 \\
8 \\
\end{array}$ & 1 & & $\begin{array}{c}86.1 \\
3 \\
\end{array}$ & 1 & & $\begin{array}{c}23.8 \\
1 \\
\end{array}$ & 1 & \\
\hline SE & 0.39 & 0.93 & & 5.49 & 0.49 & & 1.21 & 0.29 & & 0.40 & 0.13 & \\
\hline
\end{tabular}

The same letters in each column, on the basis of Duncan test have no significant differences at $5 \%$ level.

\section{Conclusion}

This information are of great importance for peanut breeders to choose a suitable genotype for fluctuating environments, i.e favorable or less favorable environments as well as to be cultivated under wide range of environments. Among the cultivars used in this study, genotypes Sohag nos. 116, 119 and 120 showed high mean performance for most studied characters, indicating stability across the environments and therefore, they could be used in a breeding programme for the development of high yielding stable genotypes across environments in the future 


\section{References}

Abd El-Rahman Rehab, H.A., A.A. AboEl-Ezz and Fadia H.A. Ahmed (2016) Phenotypic stability analysis for pod yield and its components in some peanut genotypes Egypt $\mathrm{J}$. Plant Breed.20 (3):639-651.

Abo Elezz, A.A. and Ghada B. Abd Elazees (2010). Stability of selected peanut breeding lines (Arachis hypogaea L.) in Upper Egypt. Egypt. J. of Appl. Sci., 25 (8B): 390-399.

ChuniLal, R., A.L., Rathnakumar, K., Hariprasanna, H. K. Gor, and B.M., Chikani, (2006). Early maturing groundnut advanced breeding lines with high day productivity under rainfed situations. E-Journal. Icrisat. Org, 5(1): 4.

Duncan, D.B. (1955). Multiple ranges and multiple F test. Biometrics. 11:1-42.

Eberhart, S.A. and W. A. Russell (1966). Stability parameters for comparing varieties. Crop Sci., 6: $36-40$

Habib, A.F., H.L., Nadaf, G. K. Kulkarni, and S.D. Nadiger, (1986). Stability analysis of pod yield in bunch groundnut. Journal of Oilseeds Research, 3: 46-50.

Hasan.Khan, Vinay S. Patted, M, B. Arunkumar and I. Shankergoud (2018). Stability Estimates for Pod Yield and Its Component Traits in Groundnut (Arachis hypogaea L.) under Farmer's Participatory Varietal Selection Int. J. Curr. Microbiol. App. Sci 7(1): 3171-3179

Janila, P., S. M., Nigam, M., Pandey, P.Nagesh, and R. K. Varshney, (2013). Groundnut improvement: use of genetic and genomic tools. Frontiers in plant science, 4:23.

Finlay, H.W. and G.N. Wilkinson (1963). The analysis of adaptation in plant breeding program. Aust. J. Agric. Res., 14: 742-754.

Joshi, H.J., G. B. Vekaria, and D. R., Mehta, (2003). Stability analysis for morphophysiological traits in groundnut. Legume Research, 26(1): 20-23.

Knauft, D.A. and D.W. Gorbet (1993). Consistency of rank correlations of peanut breeding lines for market grade characteristics and yield. Crop Sci., 33: 697-699.
Keshavarz S., M. Mesbah, Z. Ranji, R and Amiri (2001). Study on stability parameters for determining the adaptation of sugar beet commercial varieties in different areas of IRAN. J. of Sugar Beet. 17(1): 15-36.

Mahasi,M.J, R.S. Pathak and F.N. Waweru, (2006). Genotype by environment (GXE) interaction and stability in safflower (Carthamus tinctorious L.). Asian Journal of Plant Sciences 5(6): 1017-1021.

Minde A. S., M. S. Kambleand R. M. Pawar (2017). Stability analysis for pod yield and its component traits in groundnut (Arachis hypogaea L.) Asian Journal of Bio Science(12):15-20

Minimol, J.S., S.B. Datke, S.N. Deshmukh and G.N. Satpute (2001). Genotype x environment interaction in groundnut (Arachis hypogaea L.). Ann. Pl. Physio. 14 (1):74-79.

Patil, A. S., H. R., Nandawar, A. A. Punewar, and K. P. Shah, (2014). Stability for yield and its component traits in groundnut (Arachis hypogaea L.). International J. of Bio-resource and Stress Management, 5(2):240- 245.

Pradhan, K., P. K. Das, and R. K. Patra, (2010). Genotype $\times$ environment interaction for pod yield and components of groundnut varieties in warm sub-humid climate and moderately acidic soil. Indian Journal of Genetics, 70(2): 201-203.

Senapati, B. K., D. Maity, and G. Sarkar, (2004). Stability evaluation of summer groundnut (Arachis hypogaea L.) under coastal saline zone of West Bengal. Legume Research, 27(2): 103106.

Steel,R.G.D., J.H.Torrie, and D.A Dicky (1997). Principles and Procedures of Statistics. A biometrical Approach. $3^{\text {rd }}$ ed. McGraw Hill inc. New York, U.S.A

Thaware, B. L. (2009). Stability analysis for dry pod yield in Spanish bunch groundnut. Agric. Sci. Digest, 29(3): 221-223.

Zerihun J, A, Amsalu and F. Fekadu, (2011). Assessment of yield stability and disease responses in Ethiopian Barley (Hordeium vulgare L.) landraces and crosses. Int. J. Agric. Res. 6:754-768 


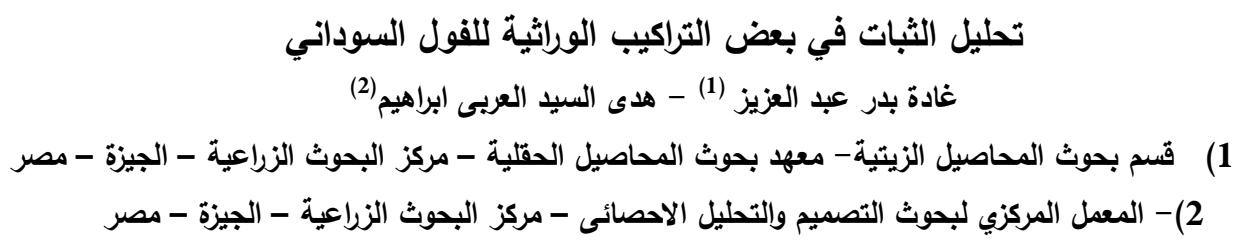

تمت زراعة ثمانية تراكيب وراثية من الفول السوداني في موقعين خلال أربعة مواسم صيفية متتالية من 2014 إلى 2017 لإعطاء ثمانية

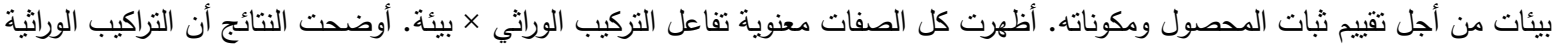

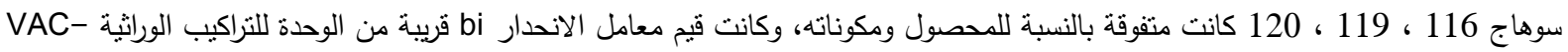

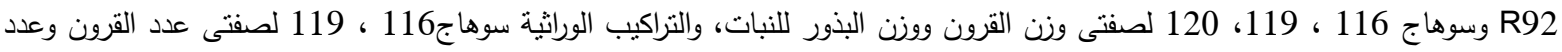

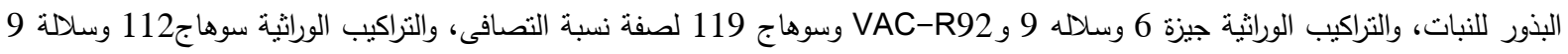

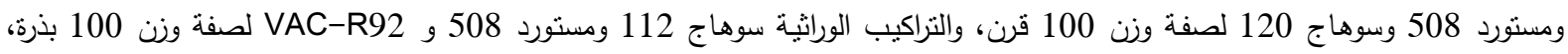

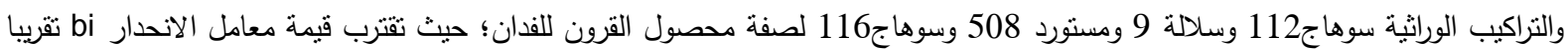

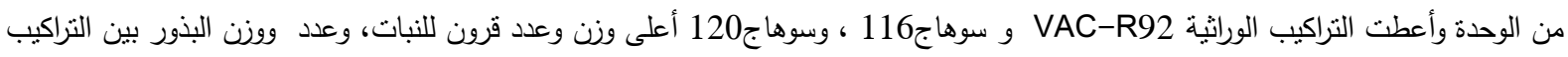

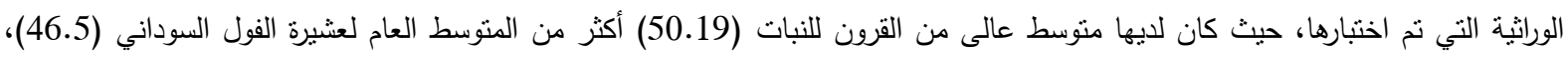

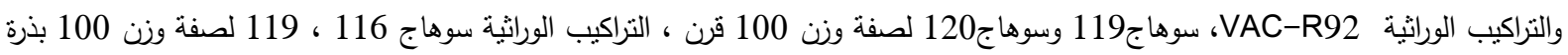

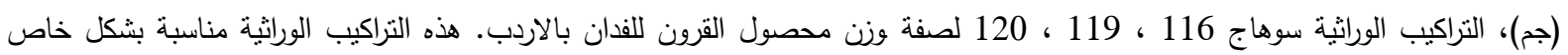

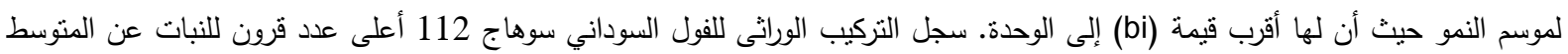

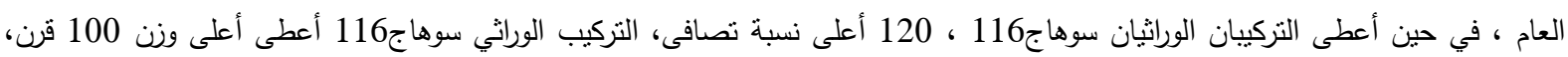

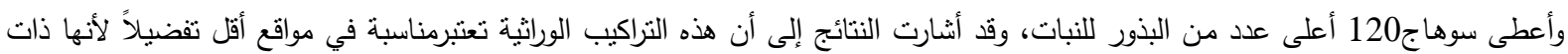

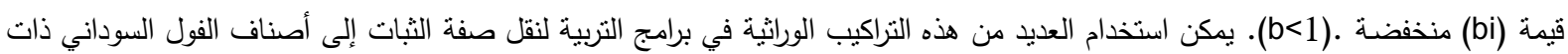
الإنتاجية العالية متل الثراكيب الوراثي سوهاج 120 والذي كان أفضلها. 